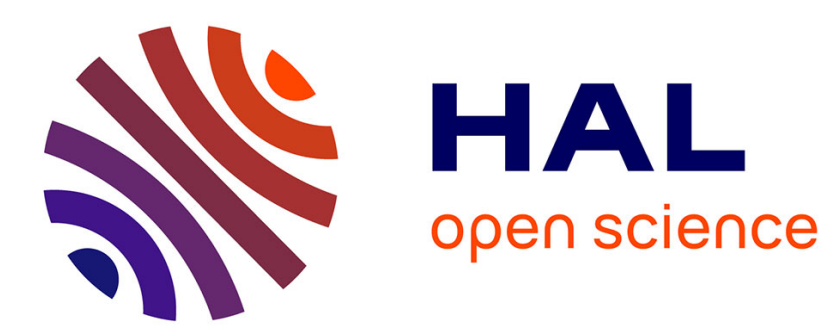

\title{
Réflectométrie à angle variable pour l'étude des couches adsorbées
}

\author{
P. Schaaf, Ph. Déjardin, Anne Schmitt
}

\section{To cite this version:}

P. Schaaf, Ph. Déjardin, Anne Schmitt. Réflectométrie à angle variable pour l'étude des couches adsorbées. Revue de Physique Appliquée, 1986, 21 (11), pp.741-745. 10.1051/rphysap:019860021011074100 . jpa-00245495

\section{HAL Id: jpa-00245495 https://hal.science/jpa-00245495}

Submitted on 1 Jan 1986

HAL is a multi-disciplinary open access archive for the deposit and dissemination of scientific research documents, whether they are published or not. The documents may come from teaching and research institutions in France or abroad, or from public or private research centers.
L'archive ouverte pluridisciplinaire HAL, est destinée au dépôt et à la diffusion de documents scientifiques de niveau recherche, publiés ou non, émanant des établissements d'enseignement et de recherche français ou étrangers, des laboratoires publics ou privés. 


\title{
Réflectométrie à angle variable pour l'étude des couches adsorbées
}

\author{
P. Schaaf, Ph. Déjardin et A. Schmitt \\ Institut Charles Sadron (CRM-EAHP) CNRS-ULP, 6, rue Boussingault, 67083 Strasbourg Cedex, France
}

(Reçu le 18 novembre 1985, révisé les 18 avril et 11 juillet 1986, accepté le 29 juillet 1986)

\begin{abstract}
Résumé. - Nous décrivons la mise en œuvre d'un réflectomètre à angle variable permettant d'explorer avec une grande précision angulaire les variations de l'intensité réfléchie pour une onde de type « $\mathrm{p}$ », au voisinage immédiat de l'angle de Brewster. En appliquant cette technique à l'étude d'une couche adsorbée et saturée de fibrinogène, il apparaît que le modèle d'une couche homogène et uniforme caractérisée par son épaisseur et son indice est une bonne approximation.
\end{abstract}

\begin{abstract}
We describe the development of a variable angle reflectometer allowing a precise angular exploration of the reflected beam intensity for a « $p$ " wave, in the close vicinity of the Brewster angle. Application of this technique to the study of a saturated adsorbed fibrinogen layer shows that the model of an homogeneous and uniform interfacial layer characterized by its thickness and its refractive index provides a good structural approximation.
\end{abstract}

\section{Introduction.}

L'étude des macromolécules synthétiques et biologiques adsorbées à l'interface solide/liquide a connu un grand essor depuis 20 ans. Les raisons en sont multiples, et l'on peut citer le développement de nouveaux concepts théoriques [1], celui de nombreuses applications potentielles ainsi que le perfectionnement des techniques d'investigation. Nous avons développé, après d'autres chercheurs [2-4], les méthodes de marquage radioactif [5-6] et la mesure d'épaisseurs hydrodynamiques de couches adsorbées [7-9]. Parmi les techniques optiques, l'ellipsométrie a suscité le plus grand nombre de travaux; elle permet de déterminer l'épaisseur et l'indice d'une couche homogène équivalente à la couche adsorbée.

Pour étudier plus finement la structure des couches adsorbées, nous mettons actuellement en œuvre la réflectométrie. Cette technique a été récemment utilisée à un angle d'incidence donné (voisin de l'angle de Brewster) pour étudier la cinétique d'adsorption de protéines sur des surfaces solides [10]. Les possibilités et limites de cette méthode optique ont été analysées dans un précédent article [11]. Il en ressort qu'avec des profils de concentration interfaciaux de faible épaisseur (inférieure ou de l'ordre de $100 \AA$ Å), seule l'étude de la réflectivité des ondes de type $p$ (polarisation dans le plan d'incidence) au voisinage de l'angle de Brewster peut conduire à des informations sur la structure de l'interface. Bien que ce résultat ait été publié pour des profils sigmoïdaux, nous avons vérifié qu'il reste valable pour une structure de couche adsorbée.
C'est pourquoi nous avons réalisé un réflectomètre à angle d'incidence variable. Après avoir décrit le dispositif expérimental et les procédures de mise au point, nous présenterons quelques résultats expérimentaux. Ils ont été obtenus avant et après adsorption sur la silice d'une protéine du plasma sanguin, le fibrinogène. La caractérisation statique et dynamique de cette couche adsorbée a fait l'objet de plusieurs travaux dans notre laboratoire $[6,7,12]$ et dans d'autres équipes [13, 14]. Aussi pourrons-nous confronter nos résultats à ceux obtenus par d'autres techniques, notamment l'ellipsométrie [15-17].

\section{Montage expérimental.}

Le dispositif expérimental est représenté schématiquement sur la figure 1 . Nous avons utilisé un laser hélium-néon polarisé (Melles-Griot ref. : 05LPH151), d'une puissance de $5 \mathrm{~mW}$. Le faisceau de lumière émis par le laser est focalisé sur la surface à étudier à l'aide d'une lentille cylindrique $L$ de distance focale $f=40 \mathrm{~cm}$ (Micro-contrôle ref.: 5318811). Un premier polariseur P1 permet d'améliorer la qualité de la polarisation du faisceau incident et de bien sélectionner la polarisation dans le plan d'incidence. Le faisceau lumineux se réfléchit ensuite sur la surface $S$ constituée par la face plane (travaillée optiquement) d'un demi-cylindre plein. Cette géométrie particulière de la cellule permet aux faisceaux incident ou réfléchi de toujours pénétrer ou émerger normalement à la partie cylindrique de la cellule $\mathrm{C}$. La focalisation du faisceau laser sur la surface plane de la cellule (plus précisément sur l'axe 


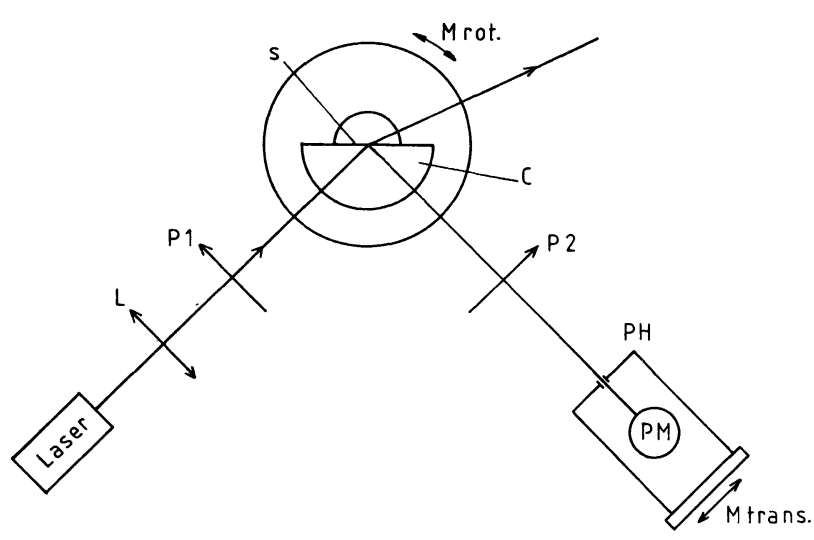

Fig. 1. - Schéma du réflectomètre à angle variable. $\mathrm{L}$ : lentille convergente ; P1 et $\mathrm{P} 2$ : polariseurs ; $\mathrm{C}$ : cellule de mesure ; $\mathrm{S}$ : surface réfléchissante ; $\mathrm{PH}$ : trou microscopique de $100 \mu \mathrm{m}$ de diamètre ; PM : photomultiplicateur ; $\mathbf{M}_{\text {trans }}$ : platine de translation micrométrique ; $\mathbf{M}_{\text {rot }}$ : platine de rotation micrométrique.

[Diagram of the variable angle reflectometer. $\mathrm{L}:$ focusing lens ; P1 and P2 : polarizers ; $\mathrm{C}$ : experimental cell ; S : reflecting surface ; $\mathrm{PH}$ : pin-hole of $100 \mu \mathrm{m}$ diameter ; PM : photomultiplier ; $\mathbf{M}_{\text {trans }}$ : microscopic translation device $; \mathbf{M}_{\mathrm{rot}}:$ microscopic rotation device.]

du demi-cylindre) et la forme particulière de la cellule permettent de nous affranchir de la divergence du faisceau laser. On évite ainsi des corrections angulaires fastidieuses. La lumière réfléchie traverse ensuite un deuxième polariseur P2. Celui-ci est réglé de manière à resélectionner l'onde $\mathrm{p}$. On réduit ainsi les erreurs introduites par l'imperfection des deux polariseurs et le défaut de réglage du polariseur P1. Les deux polariseurs P1 et P2 (MellesGriot ref. : 03FPG001) ont un coefficient d'extinction de $10^{-5}$. On atténue de plus l'intensité de la lumière diffusée par la cellule. Un trou microscopique $\mathrm{PH}$ de 100 microns de diamètre permet d'obtenir une bonne sélectivité angulaire dans les mesures. L'intensité du faisceau réfléchi est enfin mesurée à l'aide d'un photomultiplicateur (PM) de marque Hamamatsu (ref. : RS928).

On effectue les réglages à l'aide des deux platines micrométriques Mrot et Mtrans. Avec la platine Mrot (platine de rotation Micro-contrôle ref. : TR809) on modifie l'angle d'incidence en changeant l'inclinaison de la surface $S$. La rotation s'effectue autour de l'axe du cylindre de la cellule. La platine Mtrans (Micro-contrôle ref. : MR8025) permet de translater le PM ainsi que le trou microscopique pour placer le détecteur à la position correspondant à l'angle de réflexion du faisceau.

Pour mesurer l'intensité réfléchie à un angle d'incidence donné, on place le PM à la position adéquate à l'aide de Mtrans. On recherche ensuite le maximum de signal en réglant l'inclinaison de la cellule à l'aide de Mrot. Vu la taille du trou microscopique $(100 \mu \mathrm{m})$ et la distance $R$ à la surface plane de la cellule $(500 \mathrm{~mm})$, l'intensité mesurée par le PM correspond à une demi-largeur angulaire de $(\mathrm{e} / 2 \mathrm{R})$ soit $0,006 \mathrm{deg}$.

\section{Procédure expérimentale.}

La procédure expérimentale que nous avons adoptée est la suivante :

(i) La cellule étant enlevée, on aligne d'abord les deux bras du réflectomètre pour repérer les orientations angulaires des polariseurs croisés.

(ii) On remplit la cellule avec le solvant.

(iii) On se place près de l'angle de Brewster, le polariseur P2 étant orienté de manière approchée perpendiculairement au plan d'incidence. On détermine alors la position angulaire du polariseur P1 (autour du plan d'incidence) qui minimise le signal. Dans cette position le polariseur P1 ne transmet que les ondes polarisées dans le plan d'incidence.

(iv) La position du polariseur $\mathrm{P} 2$ se déduit de celle de $\mathrm{P} 1$ en rendant $\mathrm{P} 2$ parallèle à $\mathrm{P} 1$, à l'aide des repères pris à l'étape (i).

(v) On détermine l'intensité réfléchie $I_{\exp }(\theta)$ pour différents angles d'incidence $\theta=\theta_{\mathrm{B}}+\Delta \theta$ autour de l'angle de Brewster $\theta_{\mathrm{B}}$. Si la réflexion obéit aux lois de Fresnel, l'intensité mesurée doit être de la forme :

$$
I_{\mathrm{th}}=I_{0}+A \times R_{\mathrm{F}}\left(\theta_{\mathrm{B}}+\Delta \theta\right)
$$

$I_{0}$ correspond au bruit de fond du PM ainsi qu'à la diffusion de la lumière par la cellule. Ce paramètre est indépendant de l'angle d'incidence, dans le domaine exploré. Le paramètre $A$ correspond à la constante de l'appareil. La quantité $\left(I_{0}+A\right)$ équivaut au signal mesuré en cas de réflexion totale sur la surface $S . \quad \theta_{\mathrm{B}}$ est déterminé par les indices des 2 inilieux extrêmes et $\Delta \theta$ est donné par la relation $\Delta \theta=\left(X-X_{\mathrm{B}}\right) /(2 \times R) . X$ représente l'abscisse de la position du PM sur l'axe de Mtrans et $X_{\mathrm{B}}$ correspond à la position du PM pour l'angle de Brewster. Le paramètre $X_{\mathrm{B}}$ doit être introduit du fait que toutes les mesures des angles d'incidences sont des mesures relatives à une position donnée de l'appareil. La fonction $R_{\mathrm{F}}(\theta), A$ et $X_{\mathrm{B}}$ sont obtenues par une méthode classique de moindres carrés, en minimisant la quantité :

$$
S=\sum_{i}\left(I_{\operatorname{cxp}}\left(X_{i}\right)-I_{\text {th }}\left(I_{0}, A, X_{\mathrm{B}}, X_{i}\right)\right)^{2}
$$

par rapport à ces trois paramètres. La relation (1) sert en quelque sorte de ligne de base pour les points expérimentaux déterminés après adsorption. Typiquement nous avons obtenu un bruit de fond $I_{0}=6,33 \times 10^{-3} \mathrm{mV}$ et une constante $A=1,768 \times$ $10^{7} \mathrm{mV}$. La réflectivité $R(\theta)$ est définie par la relation :

$$
R(\theta)=\left(I_{\exp }(\theta)-I_{0}\right) / A .
$$

(vi) Après remplissage de la cellule de mesure avec la solution de protéine et obtention d'un quasiéquilibre d'adsorption, la cellule est rincée avec le solvant pur. La courbe de réflectivité expérimentale 
obtenue après adsorption est comparée à la courbe de réflectivité du solvant pur avant adsorption et analysée en tenant compte des valeurs des paramètres $I_{0}, A$ et $B$ déterminés avant adsorption.

Nous avons appliqué cette méthode à l'étude d'une couche dense de fibrinogène adsorbé sur de la silice. La protéine utilisée était du fibrinogène humain (grade $L$ ) de la Société Kabi que l'on dissout dans du tris(hydroxyaminométhane) à raison de $50 \mathrm{mg}$ de fibrinogène pour $100 \mathrm{~g}$ de solution tampon à $\mathrm{pH} 7,35$. La solution est dépoussiérée par centrifugation à $17300 \mathrm{~g}$ pendant $45 \mathrm{~min}$.

Avant chaque expérience la surface de silice est nettoyée au mélange sulfo-chromique afin d'éliminer toutes les matières organiques. Cette surface est ensuite rincée abondamment à l'eau déionisée et filtrée (Système Super-Q Millipore avec un filtre de $0,2 \mu \mathrm{m})$. Après avoir rempli la cellule avec la solution tampon pure, on déplace celle-ci par la solution de protéine. Après environ $60 \mathrm{~min}$, la solution de fibrinogène est remplacée par la solution tampon ce qui évite de mesurer dans le signal une éventuelle contribution due au changement d'indice de la solution de fibrinogène par rapport à la solution tampon pure.

\section{Résultats expérimentaux et discussion.}

Les variations de la réflectivité $R(\theta)$ relative à l'interface silice/solution tampon sont portées sur la figure 2 en fonction de l'angle d'incidence, au voisinage de l'incidence brewstérienne. Cette courbe expérimentale est ajustée à la courbe théorique, selon les équations (1) et (2). On observe un bon accord entre prévision théorique et données expérimentales, et la précision sur ces dernières est très satisfaisante.

Pour préparer la couche de fibrinogène adsorbé, il est possible de suivre la cinétique d'adsorption en se plaçant à un angle d'incidence très proche de l'angle de Brewster. La courbe de la figure 3 illustre les variations de $R(\theta)\left(\theta \approx \theta_{\mathrm{B}}\right)$ en fonction du temps. La partie $\mathrm{A}$ de cette courbe correspond à une adsorption rapide du fibrinogène sur la silice. $\mathrm{La}$ durée de cette phase est d'environ $20 \mathrm{~min}$. Elle est suivie d'une augmentation beaucoup plus lente et quasi linéaire de la réflectivité. Ce résultat est à rapprocher avec l'augmentation lente de l'épaisseur (à indice constant) de la couche adsorbée observée par Cuypers [15]. Ceci correspond donc à une adsorption lente du fibrinogène qui peut s'accompagner éventuellement d'un réarrangement de la couche adsorbée. On remplace ensuite la solution de fibrinogène par du tris pur (partie C). On observe une diminution de la réflectivité sur une période de l'ordre de $10 \mathrm{~min}$ qui peut s'expliquer par une faible désorption du fibrinogène.

La figure 2 présente aussi les variations de réflectivité avec l'angle d'incidence en présence de la couche protéique interfaciale. On observe que le signal réfléchi est affecté de manière significative par la présence des molécules adsorbées.

Nous avons tenté d'ajuster notre courbe de réflec-

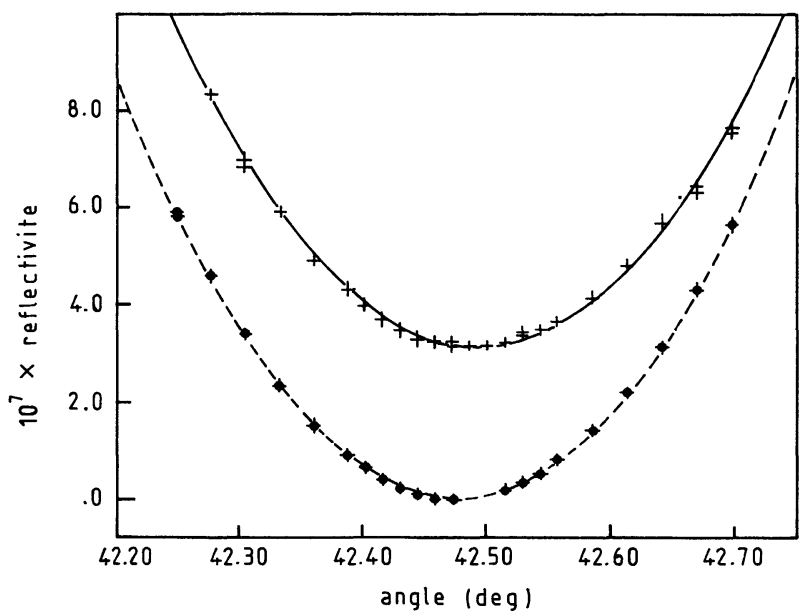

Fig. 2. - Variations de la réflectivité $R(\theta)$ pour une onde de type « $p$ » en fonction de l'angle d'incidence (exprimé en degrés) (๑) valeurs expérimentales relatives à l'interface solvant pur/silice ; $(+)$ : valeurs expérimentales après absorption d'une couche de fibrinogène ; (----) : valeurs théoriques calculées selon l'équation (1) en utilisant les formules de Fresnel (les indices des milieux extrêmes sont : 1,333 pour la solution de tris et 1,457 pour la silice), ( considérant un modèle de couche uniforme d'épaisseur $218 \AA$ et d'indice 1,360 .

[Variations of the reflectivity $R(\theta)$ for a « $\mathrm{p}$ » wave as a function of the incidence angle (expressed in degrees); (๑) experimental values related to the solvent/silica interface $;(+)$ experimental values after adsorption of a fibrinogen layer ; (---) theoretical values calculated from equation (1) using the Fresnel formulas (refractive indexes of the homogeneous media : 1.333 for the buffer solution and 1.457 for silica) ; ( $\longrightarrow$ ) theoretical values calculated for the model of an homogeneous layer of $218 \AA$ thickness and a reflective index of 1.360.]

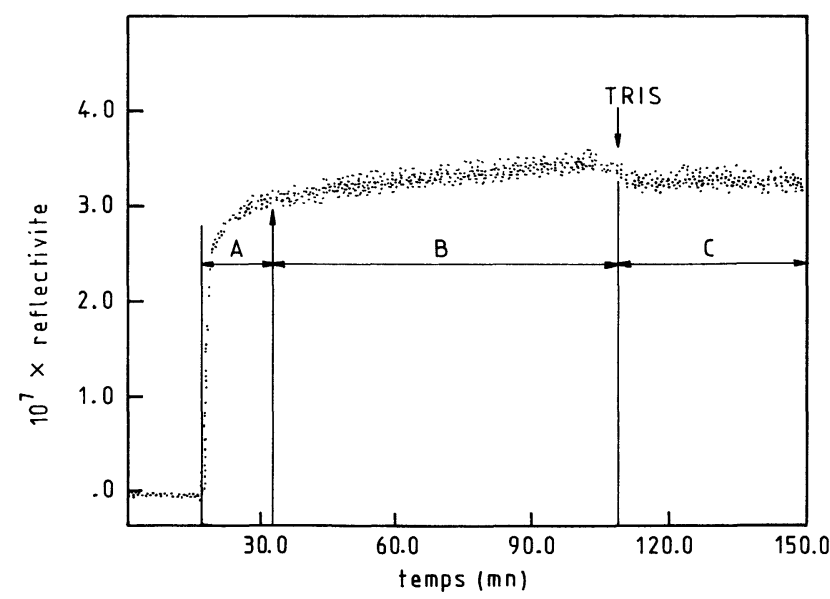

Fig. 3. - Variation de la réflectivité pour une onde " $\mathrm{p}$ 》 en fonction du temps (en min) lors du processus d'adsorption pour un angle voisin de l'angle de Brewster.

[Variations of reflectivity for $a \ll p »$ wave against time (in min) during the adsorption process in the vicinity of the Brewster angle.] 
tivité expérimentale à celle attendue pour une couche homogène et uniforme. L'ajustement s'est fait en utilisant les formules classiques de la réflectivité d'une couche pour des ondes $\mathrm{p}$ [16]. Les paramètres calculés sont l'indice de la couche et son épaisseur. Dans un tel calcul on tient compte des paramètres de l'appareil $I_{0}$, A et $X_{\mathrm{B}}$ déterminés précédemment de manière expérimentale. Un tel calcul conduit à une épaisseur de $218 \AA$ et un indice de l'ordre de 1,360 pour la couche homogène adsorbée. La courbe de réflectivité correspondant à ces valeurs peut être comparée aux données expérimentales sur la figure 2. On voit que ce modèle rend bien compte des résultats expérimentaux. L'épaisseur relativement importante ainsi que le faible indice de la couche peuvent s'expliquer par le caractère hydrophile de la surface de silice comme l'avait déjà fait remarquer Cuypers [15]. Pour illustrer la sensibilité de la technique vis-à-vis des paramètres calculés (épaisseur et indice) nous avons tracé la figure 4, relative aux couches homogènes suivantes :

$\mathrm{C} 1$ : épaisseur $218 \AA$, indice 1,360

$\mathrm{C} 2$ : épaisseur $200 \AA$, indice 1,360

C3 : épaisseur $218 \AA$, indice 1,370 .

Ces courbes nous permettent de mieux apprécier l'influence de ces paramètres sur la réflectivité. On peut ainsi en conclure que le modèle de couche homogène décrit convenablement la structure du fibrinogène adsorbé dans la limite de la sensibilité du réflectomètre.

Dans le tableau I, nous donnons quelques valeurs d'épaisseurs et d'indices publiés dans la littérature $[15,17,18]$, obtenues par ellipsométrie. On relève la

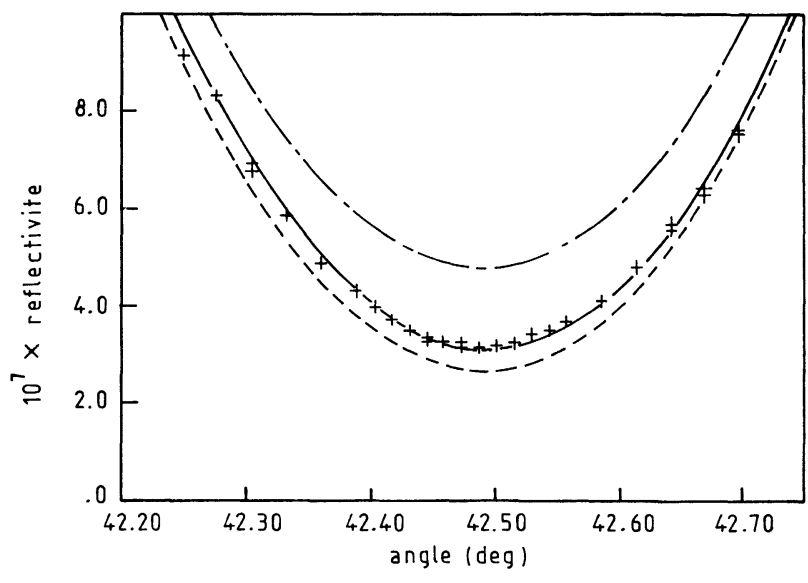

Fig. 4. - Variation de la réflectivité pour une onde " $p$ » en fonction de l'angle d'incidence (en degrés). $(+)$ : points expérimentaux après adsorption du fibrinogène ; ((voir texte) ; (--) : courbe C3 (voir texte).

[Variations of the reflectivity of $a$ « $p$ » wave as a function of the incidence angle (in degrees). $(+)$ experimental values after fibrinogen adsorption; $(\stackrel{(+)}{-}$ : curve C1 ; (---) : curve C2 ; (-- $)$ : curve C3 (see text).]

grande dispersion de ces résultats, qui peut être due à des supports d'adsorption différents.

Dans ce tableau nous avons également reporté des valeurs d'épaisseurs obtenues par des mesures hydrodynamiques [7]. Celles-ci sont nettement plus importantes que toutes celles mesurées par les méthodes optiques. Ceci peut être compris de manière qualitative à partir de la théorie de Déjardin et Varoqui [19] portant sur l'interprétation des épaisseurs hydro-

Tableau I. - Epaisseur et indice de réfraction caractéristiques de couches de fibrinogène adsorbées sur différents supports solides, dans l'hypothèse d'un modèle de couche homogène, et déterminés par différents auteurs.

[Thickness and refractive index characterizing adsorbed fibrinogen layers on various solid adsorbents, for the model of an homogeneous layer as obtained by different authors.]

\begin{tabular}{|c|c|c|c|c|c|}
\hline Références & $\begin{array}{l}\text { Epaisseur } \\
(\AA)\end{array}$ & $\begin{array}{l}\text { Indice de } \\
\text { réfraction }\end{array}$ & Méthode & Support & $\begin{array}{l}\text { Concentration } \\
(\mathrm{mg} / 100 \mathrm{~g})\end{array}$ \\
\hline [15] & $\begin{array}{r}35 \\
120\end{array}$ & $\begin{array}{l}1,8 \\
1,4\end{array}$ & $\begin{array}{l}\text { Ellipsométrie } \\
\text { Ellipsométrie }\end{array}$ & $\begin{array}{l}\mathrm{CrO}_{2} \\
\mathrm{Cr}\end{array}$ & $\begin{array}{l}1 \\
1\end{array}$ \\
\hline [18] & 18 & & Ellipsométrie & $\mathrm{Au}$ & 20 \\
\hline$[17]$ & $\begin{array}{l}72 \\
40\end{array}$ & $\begin{array}{l}1,65 \\
1,65\end{array}$ & $\begin{array}{l}\text { Ellipsométrie } \\
\text { Ellipsométrie }\end{array}$ & $\begin{array}{l}\mathrm{Cr} \\
\mathrm{Cr}\end{array}$ & $\begin{array}{r}100 \\
10\end{array}$ \\
\hline$\left[{ }^{*}\right]$ & 218 & 1,360 & Réflectométrie & $\mathrm{SiO}_{2}$ & 50 \\
\hline [7] & $\begin{array}{l}500-600 \\
300\end{array}$ & & $\begin{array}{l}\text { Hydrodynamique } \\
\text { Hydrodynamique }\end{array}$ & $\begin{array}{l}\text { Pyrex } \\
\text { Pyrex }\end{array}$ & $\begin{array}{r}50 \\
1\end{array}$ \\
\hline
\end{tabular}

[*] Résultat relatif à notre travail.

[*] Results related to the present work. 
dynamiques. La technique hydrodynamique est très sensible aux chaînes de la protéine qui émergent dans le solvant, créant ainsi une importante contribution à la friction hydrodynamique. Par contre, comme la densité de matière provenant de ces boucles est faible, celle-ci n'affecte pas notablement l'indice local de la solution, grandeur à laquelle sont sensibles les méthodes optiques. La réflectométrie et l'ellipsométrie ne nous informent donc que sur la partie dense (donc de fort indice) de la couche adsorbée. Elles constituent ainsi des méthodes d'investigations complémentaires des mesures hydrodynamiques pour l'étude de la structure des couches adsorbées.

\section{Conclusion.}

Le but du présent article était d'illustrer, par quelques données expérimentales, les possibilités de la technique de réflectométrie pour l'étude des couches adsorbées. Ainsi, nous avons déterminé les varia- tions de la réflectivité avec l'angle d'incidence au voisinage de l'angle de Brewster, avant et après adsorption d'une couche macromoléculaire. On montre en particulier que le modèle de la couche homogène et uniforme est satisfaisant dans le cas du fibrinogène.

Notre effort actuel porte sur l'amélioration de la sensibilité de notre technique et de l'exploitation adéquate de la courbe de réflectivité, afin de pouvoir analyser l'adsorption de macromolécules synthétiques sur la silice.

\section{Remerciements.}

Ce travail a été financé par le CNRS, dans le cadre de l'ATP « Matériaux » et du GRECO « Polymères Hémocompatibles ». Nous remercions M. le Professeur H. Benoît pour son aide et ses encouragements dans le lancement de ce projet. MM. G. Maennel et F. Woehl nous ont aidé avec efficacité dans la réalisation technique de l'appareillage.

\section{Bibliographie}

[1] De Gennes, P. G., Scaling Concepts in Polymer Physics (Ithaca and New York, Cornell Univ. Press) 1971.

[2] Stromberg, R. R., Grant, W. H. et Passaglia, E., J. Res. NBS 68A (1964) 391.

[3] Rowland, F. W. et Eirich, F. R., J. Polym. Sci. A1 4 (1966) 2033 ; ibid. 4 (1966) 2401.

[4] Gramain, Ph., Myard, Ph., J. Colloid Interface Sci. 84 (1981) 114.

[5] Pefferkorn, E., Carroy, A. et Varoqui, R., $J$. Polym. Sci. Polymer Phys. Ed. 23 (1985) XX.

[6] SChmitT, A., VAROQUi, R., UNIYAL, S., BRASH, J. L. et Pusineri, C., J. Colloid Interface Sci. 92 (1) (1983) 25.

17] De Baillou, N., Déjardin, Ph., Schmitt, A. et BRASH, J. L., J. Colloid Interface Sci. 100 (1) (1984) 167.

[8] DéJardin, Ph., Thèse Université Louis Pasteur, Strasbourg (1981).

[9] Déjardin, Ph., Toledo, C., Pefferkorn, E. et VAROQUI, R., dans Ultrafiltration Membranes, A. Cooper Ed., Polymer Sci. Technol. 13 (1980) 203, Plenum Press.
[10] Welin, S., Elwing, H., Arwin, H., Lundstrom, I. et Wikstrom, M., Anal. Chim. Acta 163 (1984) 263.

[11] SchaAf, P., Déjardin, Ph. et Schmitt, A., Revue Phys. Appl. 20 (1985) 631.

[12] Voegel, J. C., De Baillou, N., Sturm, J. et SCHMITT, A., Colloids Surfaces 10 (1984) 9.

[13] Chan, B. M. C., Brash, J. L., J. Colloid Interface Sci. 82 (1981) 217.

[14] LoK, B. K., Cheng, Y. et Robertson, C. R., J. Colloid Interface Sci. 91 (1983) 87.

[15] Cuypers, P. A., Hermens, W. T. et Hemker, H. C., Ann. N.Y. Acad. Sci. 283 (1977) 77.

[16] AzZam, R. M. A. et Bashara, N. M. dans Ellipsometry and Polarized Light (North-Holland, Amsterdam) 1977 p. 283-288.

[17] TOTh, K., VuYe, G., VAiner, H., Ronto, Gy. et Aslanian, D., J. Physique Colloq. 44 (1983) C10-167.

[18] Davis, R. B., Azzam, R. M. A. et Holtz, G., Surface Sci. 96 (1980) 539.

[19] Déjardin, Ph. et Varoqui, R., J. Chem. Phys. 75 (1981) 4115. 